\title{
Implementation of Augmented Reality System for Smartphone Advertisements
}

\author{
Young-geun Kim and Won-jung Kim \\ Department of Computer Science Sunchon National University \\ Suncheon, 540-742, Korea
}

kwj@sunchon.ac.kr

\begin{abstract}
The recent development and popularization of personal mobile devices has brought a lot of changes into information search and offering. In particular, the newly emerged augmented reality system made possible an innovative way of information acautsition by providing additional information of the virtual world to the real world. This work used the markerless augmented reality system on smartphones ta design and implement the smartphone application service aimed at efficiently conveying information on advertisements to users. The conventional advertising applications simply introduce and explain goods by inducing consumers to have an interest, whereas the markerles@application service developed in this work interacts with the augmented reafity system and database management system to quickly provide more accurate and diversifiedinformation tousers.
\end{abstract}

Keywords: Augment Reality, smartphone, Advertisements, Non-marker

\section{Introduction}

With the recent tapid penetration of mobile devices, portable smart devices have become popular. As a result, the demands of personalized services are on the rise, leading to emergence of smartphones equipped with various functions. Additionally, as the traditional augmented reality technology has been planted into mobile devices, mobile augmented reality has been distributed in the type of smartphone application, drawing more attention [1].

It is to an advantage of portable smart devices to use users' situational information according to their movements, like positioning information or sensor information, to materialize more the targets of advertisements. Thanks to expansion of mobile device users, users' friendly attitudes toward the devices as media, and the mobile devices' characteristics, the mobile advertising has recently experienced the most fierce competition in the mobile market [2]. Most conventional mobile augmented reality applications related to advertisements provide the service of augmenting Point Of Interest (POI) based on the positioning system of users and their surroundings. However, because of the margin of error of satellites, the mobile augmented reality applications have limitations of offering accurate information to users quickly [3]. Also, they fail to guarantee the accuracy and straightforwardness in terms of the connection between visible information and real information. Although augmented reality applications are interesting and fun, they have yet to develop enough to show their vision of profit creation [4].

In this work, these researchers implemented the two-way communications system between augmented reality and users through an markerless augmented reality application, rather than a positioning augmented reality application, in order to solve the margin of error that 
conventional augmented reality applications related to advertisements have and to provide accurate information for users more quickly.

In the Chapter 2 (relevant studies) of this thesis, the technologies and trends of augmented reality, the services used in the advertising area, and the overall direction of the system implemented in this work are described. In Chapter 3, the design and implementation of the system is described, and the system's usefulness and something to be improved, which were drawn from a field test, are investigated. In the last chapter, this work came to conclusions and suggested future research.

\section{Relevant Studies}

\subsection{Augmented Reality (AR)}

Augmented reality is a concept of supplementing the real world with the yirtual world. Although it uses a virtual environment created by computer graphics, its main playground is the real environment. Computer graphics serve the function of adding necessary information into the real environment. In so doing, t makes up for the weak point of unreality which can occur in the environment providing only the virtuak world. Augmented reality is to improve the recognition tools for the real world and thus to efficiently interact between humans and computers.

According to the object-tracking techniques, augmented system is divided into positioning, marker, and markerless augmented reality services. As shown in Table 1, positioning augmented reality system is the most used one thanks to the wide penetration of GPS smartphones.

Table 1. Location-based augmented reality services.

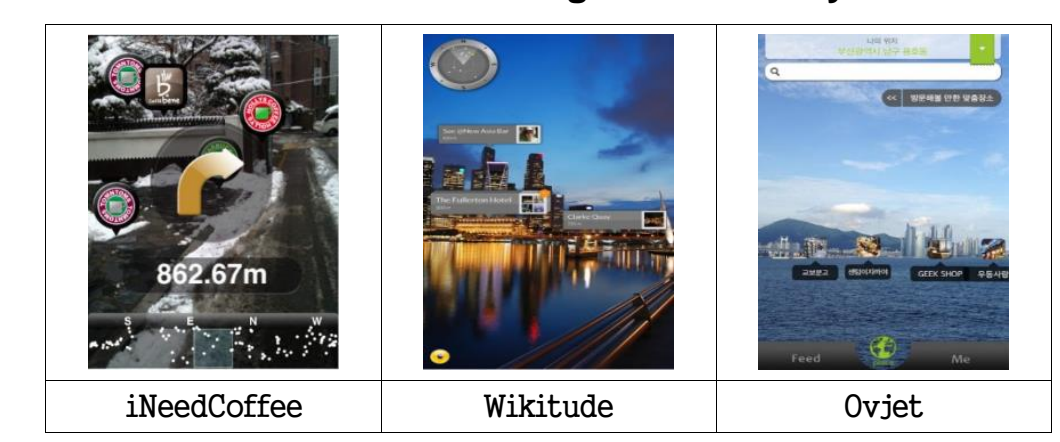

As for the positioning augmented reality system, when there are more than two contents to be displayed at the same position of a smartphone screen with a limited size, overlapping can block users from obtaining proper information. Also it does not take into account a user's possibility of actual movement, so that if a neighboring content is displayed on an obstacle which a user can't pass by, it is difficult to decide the user's actual moving path. Furthermore, since it can recognize only positions, changing information on the real world leads to updating the information on augmented reality. As a result, it does not guarantee reliability of information [5].

The marker augmented reality system uses a technique to track the markers already agreed. As shown in Figure 1, a marker usually features a square frame with white background color, and the recognized marker has three-dimensional coordinates and compounds a threedimensional object. The technique requires the previous arrangement of markers before being commercialized. So although it can smoothly be used in a space with a specific purpose, like 
museum, it faces 'social resistance' as it is applied to something focusing on design layouts, like publications. Additionally, since it uses black markers mostly, it is sensitive to lighting of the place where markers are installed, and it can be used as part of markers is covered. Therefore, it is technically urgent to supplement its disadvantages [6].

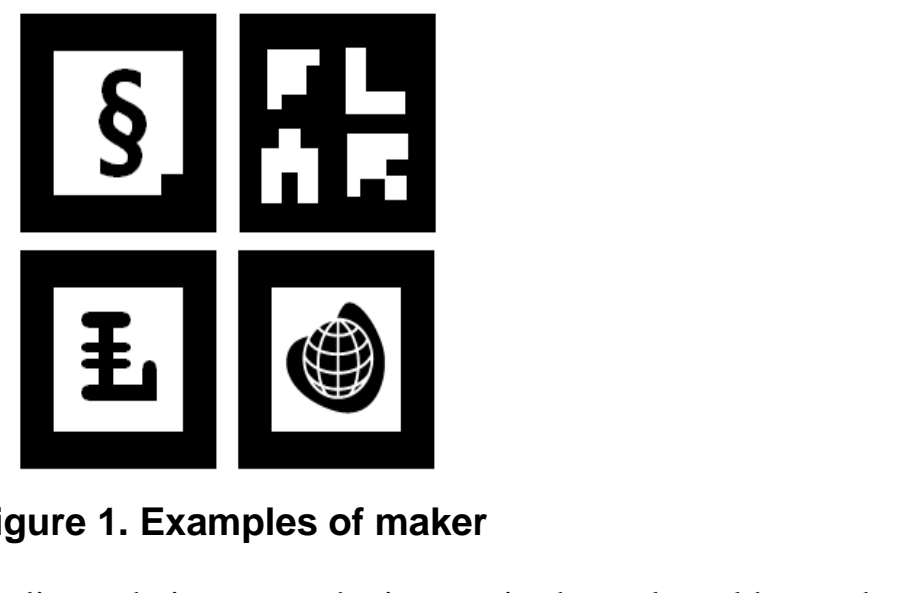

The markerless augmented reality technique uses the images in the real world to make up for the weak points of marker augmented reality system, and extracts points, lines, corners, textures, and other features, as shown in Figure 2, through camera images. It has been recognized as the most difficult and eritical technique in the augmented reality area. That is because it detects and recognizes lighting, partial oyerlapping, and directions on the basis of an object's characteristics, and shows excellentperformance [7].

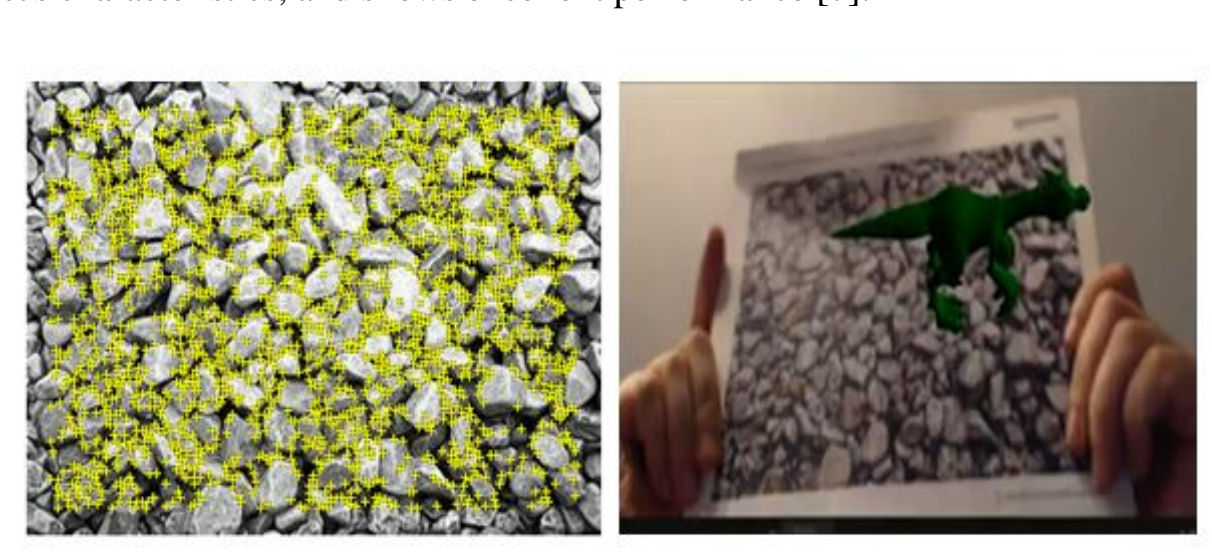

Figure 2. Examples of augmented reality Non-marker

\subsection{Augmented Reality Application Service in the Advertising Area}

For this thesis, the researchers surveyed the existing augmented reality services related to advertisements running on the smartphone application environment, analyzed their strong and weak points, and used them to set up the direction of the service to be implemented.

Advertising through smartphones is a way of conveying the information on ideas, services, or products, which are aimed at increasing profits of advertisers, to consumers with the use of the smartphone application environment. It is categorized into two types: application-making and contents exposure. The reason that today's smartphone applications draw more attention as advertising media is that the applications have the possibility to provide a customized advertising message to diverse consumers in a more effective way [8]. 
There are many advertising applications to which augmented reality system is applied. The most typical applications using positioning augmented reality system are iNeedCoffee used to locate a cafe, Wikitude used to find restaurants and people and obtain place information within a visible distance and to play games, and Ovjet which has the enhanced social functions of sharing information with neighboring users and finding places. However, the GPS based positioning augmented reality applications use the satellites for nongovernmental purposes so that they have the margin of error. Also because of the error of their digital magnetic compass used to recognize a direction, they fail to provide accurate information for users.

There are some markerless augmented reality applications. For examples, the sinartphone application created by Volkswagen augments a three-dimensional animation as it goes toward an outdoor advertisement or a bus stop advertisement. RAY App developed by Kia Motors augments a three-dimensional object through the company's own advertisement posted on newspapers. Aside from those, in developing applications, many âttempts to apply markerless augmented reality technique to advertisements are made, but do not go further than simply to trigger users' interests and curiosities.

\section{System Design}

The augmented reality system service implemented in this thesis uses markerless augmented reality technique, and has a pe smartphone application. Also, it is aimed to conveying three-dimensional models of videos to consumers through outdoor signboards or flyers. The implemented system service does nolsimply trigger users' interests through their augmented three-dimensional models, but interacts with database management system to deliver a variety of information to users quicklyand accurately. And it implemented the twoway communication services between augmented reality and consumers, such as booking service.

To implement the markerless augmented reality system, the service of this work used Vuforia SDK y2.6 of Qualcomm and Unity, a software application to develop games. As a database management system, MySQL was used. The data transmission between the smartphone augmented realityapplication and the DBMS was designed to be made by PHP and XML. The below Figure 3 illustrates the system architecture of the service to be developed in this work

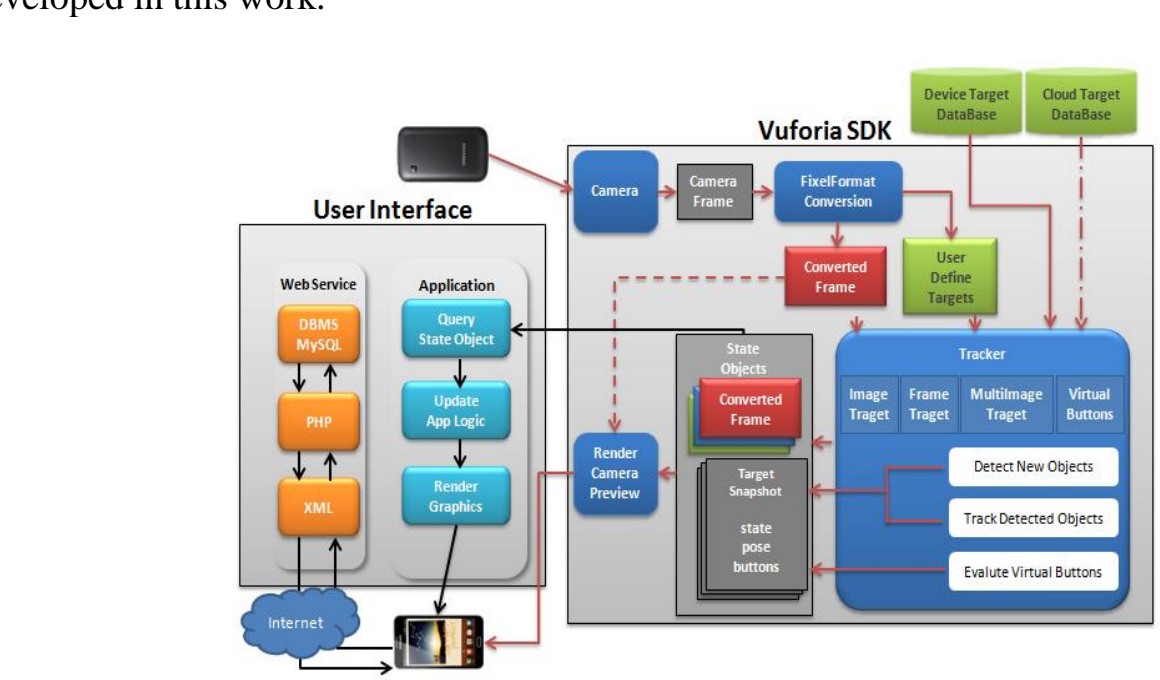

Figure 3. System Architecture 
The hardware system used to implement the augmented reality application in this work has the specs. of Intel(R) Core(TM) i3-220 CPU 3.30GHz, 8.0GB RAM, and NVIDIA GeForce GTC 550Ti. The implemented augmented reality system was tested in the following H/W \& S/W environment: CPU Dual Core 1.5GHz, Android 4.1.2 Jelly Bean, Built-in memory $16 \mathrm{~GB}$, and the resolution of $1280 * 800$.

\section{System Implementation}

The augmented reality application system developed in this work uses leaflets and outdoor signboards of stores to augment the three-dimensional model based indoor information and booking information of stores, and makes possible users' direct booking.

Once the augmented reality system decides a leaflet or an outdoor signboard to be advertised, it uploads a JPG image to Vuforia SDK v2.6 of Qualcomm and then download the matching coordinates of the extracted character point in the type of Unity Package, as shown in Figure 4 below.

To make an augmented reality application by Unity, the system imports the Unity Package downloaded as the matehing coordinates of Vuforia SD's vuforia-android and vuforiaimagetargets-android, registers it in Hierarchy, register a three-dimensional object in ImageTarget Hierarchy as shown in Figure 5, and matches the three-dimensional object fitting a marker
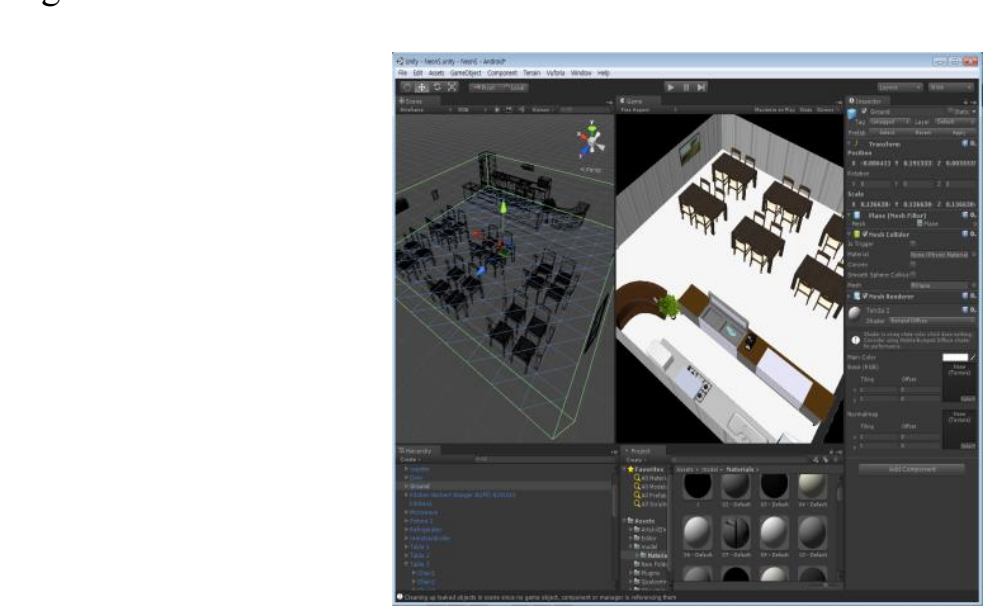

Figure 5. Matching three-dimensional objects in marker 
When an advertising image matches an three-dimensional object, only the threedimensional object is augmented. In this case, users' understanding and interests can lower, and therefore the system provides user interface, selling goods, or the function of converting the data in DBMS into an XML data if a seat is chosen, to improve users' convenience and diversity of importation.

The augmented reality application created in Unity sets up the basic environment necessary for Build, and is executed after being built in a smartphone through USB. Figure 6 illustrates the augmentation of the interior related to an advertising image running on a smartphone, and the information on selling products and seats.

\section{Conclusions}

Figure 6. Matching three-dimensional objects in marker

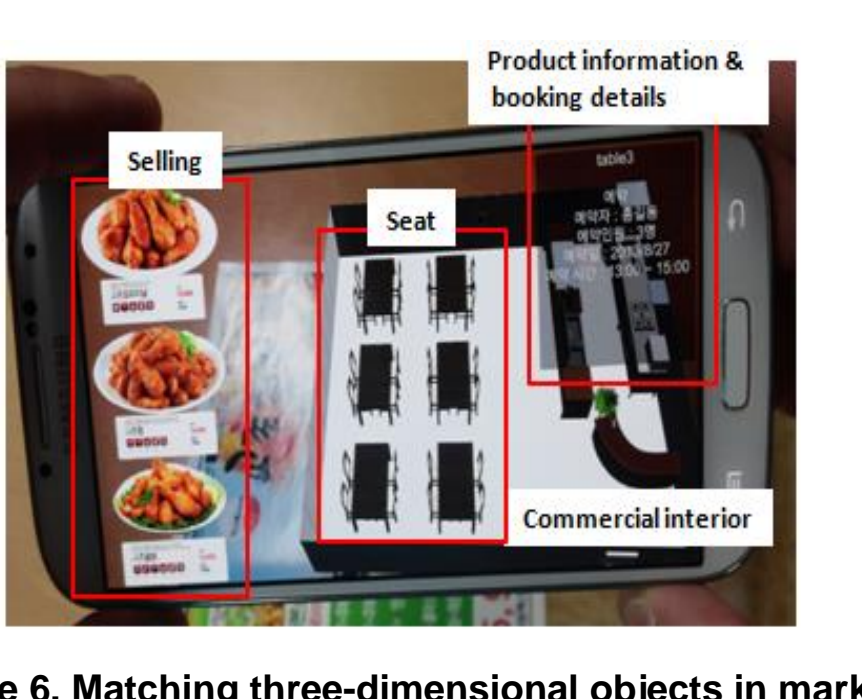

In this work, based on markerless augmented reality system technique, the augmented reality application servige for smartphones was designed and implemented to effectively convey the information of advertisements. As a result, the service was able to solve the problems that the positioning augmented reality service used in most augmented reality applications for smartphone has, and to provide a variety of information in a more accurate and fasteryay. Also the implemented system was able to not only augment three-dimensional objects in the augmented reality, but also interact with database management system to make possible two-way communication services like booking system.

Therefore, unlike the conventional advertising applications which simply introduce and explain products by triggering users' interests, the implemented system service is expected to be used in a wide range of areas including advertising, education, and tourism, by making interaction between users and augmented reality system.

In the future, it is necessary to apply the augmented reality system suggested in this thesis to actual advertisements and thereby analyze its efficiency, and study the combination of markerless augmented reality system and positioning augmented reality system to provide a variety of information. 


\section{References}

[1] J. Kim, W. Cha and J. Yoo, "The Development of the Augmented Reality Techniques in a Mobile Environment and Active Utilization Plan of the Digital Design”, Korea Digital Design Council, Digital Design Studies, vol. XIV, no. 30, (2011) April.

[2] S. Cho, S. Yi and S. -b. Suh, "A Survey of User Targeted Advertising Technologies on the Mobile Devices", Korea Institute of Information Scientists and Engineers, Journal of Information Science: Information and Communication, Article 39, no. 1, (2012) February, pp.73-86.

[3] Y. -g. Kim and W. -j. Kim, "Tourist information service on research based Smart Phone Augmented Reality", The Korea Institute of Electronic Communication Sciences, Journal Spring 2013, vol. 7, no. 1, (2003) June.

[4] S. a. Jeon, "Study on UI Design of Augmented Reality Application of Smartphone Providing Local Information - Focusing on 'Layar' and 'ScanSearch'-", Major in Advertising Design, Department of Indystrial Design, Graduate School of Hong-ik University, (2010) August.

[5] J. H. Lee, Y. H. Jang and Y. J. Kwon, "An Efficient Location Based Service based on Mobjle Augmented Reality applying Street Data extracted from Digital Map”, Journal of Korea Spatial Information Society, vol. 21, no. 4, (2013), pp. 63-70.

[6] H. k. Kim, "Cataclysm augmented reality 'video-based augmented redity' of the present and the future", POPCON 2011.032, (2011) March.

[7] H. Jo and D. Kim, "Non-Marker Based Mobile Augmented Reality Technology Using Image Recognition", The Korea Institute of Signal Processing and Systems, Ins titute of Signal Processing and Systems, vol. 14, no. 4, (2011) October.

[8] J. A. Kim, "A Study on the Mobile Advertisemenf Attitude of Smartphone Users: Focused on Mobile Advertisements Using Application”, Dept. of Advertising Design Graduate school of Industry Hongik University, (2010) December.

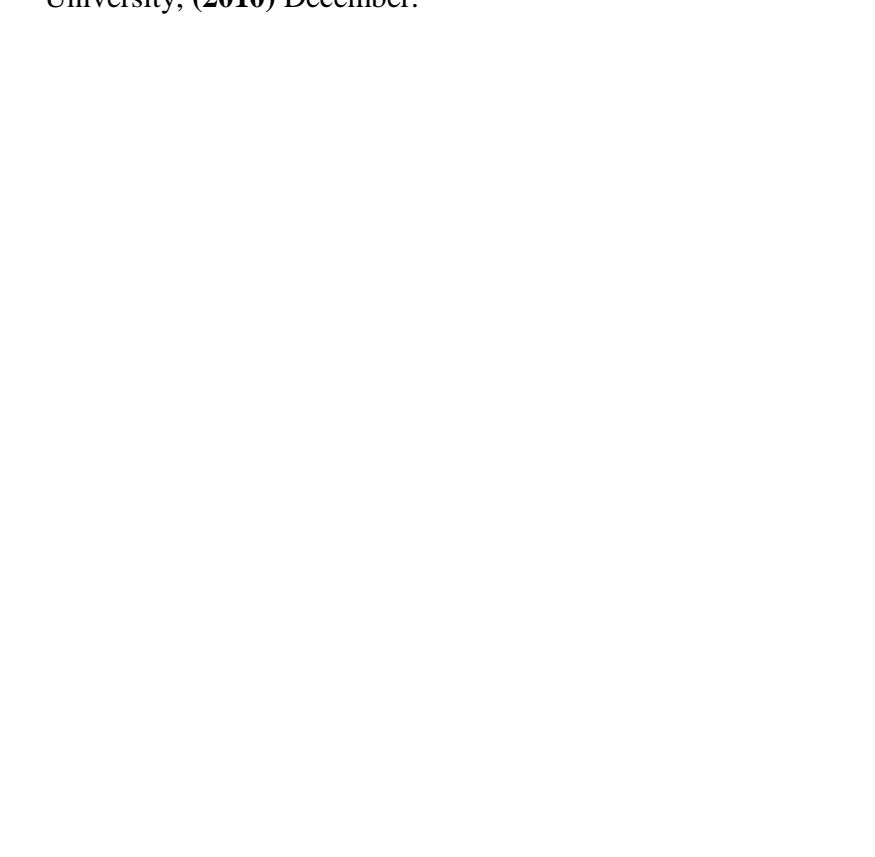


International Journal of Multimedia and Ubiquitous Engineering Vol.9, No.2 (2014)

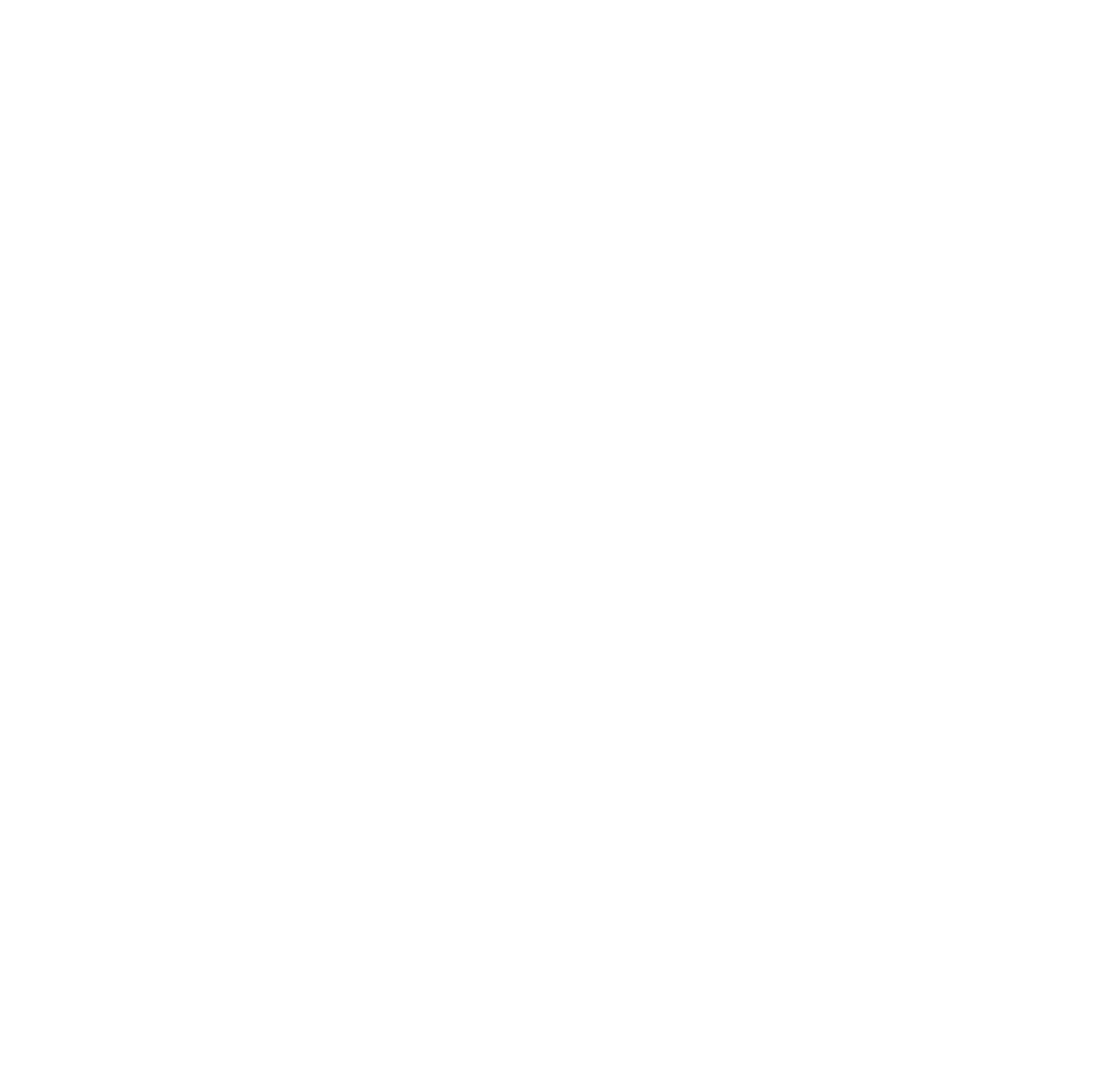

